\title{
Metodología para el análisis de vulnerabilidad en cuencas abastecedoras de agua ante la variabilidad climática'
}

\author{
Mónica Patricia Valencia Rojas \\ Apolinar Figueroa Casas ${ }^{* *}$ \\ Diana Marcela Ruiz Ordóñez ${ }^{* * *}$ \\ Juan Diego Otero Sarmiento ${ }^{* * * *}$ \\ Juan Pablo Martínez Idrobo***** \\ Victoria Eugenia Ceballos Sarria \\ Samir Carlos Joaquí Daza \\ Dayan González Quijano*******
}

Recibido: 30/09/2013 • Aceptado: 27/06/2014

\section{Resumen}

Este artículo de investigación presenta una metodología para el análisis de vulnerabilidad de fuentes abastecedoras de agua con resultados regionales (Popayán y Cajibío), considerando los siguientes elementos: 1) Incorporación de indicadores pertinentes para fuentes abastecedoras de acueductos municipales, 2) Saberes locales sobre percepción, clima e identificación de problemáticas relacionadas, 3) Procedimientos matemáticos y ajuste del algoritmo para calcular la vulnerabilidad y 4) articulación con instrumentos de planificación territorial. Como resultado, la vulnerabilidad en el escenario actual de la subcuenca río Las Piedras (Popayán) es medio baja $(0,42)$, producto de la capacidad adaptativa generada por procesos sinérgicos desarrollados entre los actores locales; en contraste, la subcuenca del río Michicao (Cajibío) presenta una vulnerabilidad media alta $(0,54)$, debido a la desarticulación social, baja presencia institucional y nula inversión ambiental.

Palabras clave: acueducto, adaptación, cambio climático, indicadores

\footnotetext{
$1 \quad$ Producto de una investigación terminada, en el marco del Convenio de cooperación celebrado entre la Corporación Autónoma Regional del Cauca (CRC) y la Corporación Centro internacional de Investigación e Innovación del Agua CIAgua N. ${ }^{\circ}$ 0201-1906-año 2013

Bióloga, Candidata a Doctor en Ciencias Ambientales, Investigadora Asociada Grupo de Estudios Ambientales (GEA), Universidad del Cauca. Email: mvalenciarojas@unicauca.edu.co

*** Doctor en Ciencias (PhD). Director Grupo GEA, Profesor titular Programa de Biología Universidad del Cauca. Email: apolinarfigueroa@unicauca.edu.co

*** Bióloga, Investigadora Asociada GEA Universidad del Cauca, Estudiante del programa de Doctorado en Ciencias Ambientales en la Universidad del Cauca. Email: dianamruiz@unicauca.edu.co

***** Biólogo, Investigador Asociado GEA Universidad del Cauca, Estudiante del programa de Doctorado en Ciencias Ambientales en la Universidad del Cauca. Email: jotero@unicauca.edu.co

Biólogo Candidato a Doctor en Ciencias Ambientales, Investigador Asociado Grupo de Estudios Ambientales, Universidad del Cauca. Email: jpmartinez@unicauca.edu.co

Bióloga, Investigadora Asociada GEA Universidad del Cauca. Email: veceballoz@unicauca.edu.co

Biólogo, Candidato a Doctor en Ciencias Ambientales, Investigador Asociado Grupo de Estudios Ambientales, Universidad del Cauca. Email: sjoaqui@unicauca.edu.co

Geógrafa, Investigadora Asociada GEA Universidad del Cauca. Email: dayan@unicauca.edu.co
} 


\title{
Methodology for the analysis of vulnerability to water watershed supplying climate variability
}

\begin{abstract}
This research paper presents a methodology for the analysis of vulnerability of water supply sources with regional results (Popayan and Cajibío), considering the following points: 1) incorporation of relevant indicators of municipal water supply sources, 2) Local knowledge on perception, and identification of climate related problems, 3) mathematical procedures and adjust the algorithm for calculating the vulnerability and 4) coordination with territorial planning instruments. As a result, the vulnerability in the current scenario of the Rio Las Piedras subbasin (Popayán) is medium low (0.42) product generated by adaptive capacity developed synergistic processes between local actors; in contrast, the basin of the river Michicao (Cajibio) has a high average vulnerability (0.54) due to social dislocation, low institutional presence and zero environmental investment.
\end{abstract}

Keywords: adaptation, climate change indicators, watershed supplying. 


\section{INTRODUCCIÓN}

El cambio climático (CC) y la variabilidad climática (VC) afectan la disponibilidad de agua en las cuencas hidrográficas [1-3] y constituyen una preocupación mundial especialmente por sus implicaciones sobre las comunidades humanas. Considerando estos efectos, se han desarrollado estudios desde diferentes enfoques para abordar la vulnerabilidad de las cuencas abastecedoras de agua integrando diferentes componentes [4]. La vulnerabilidad de las fuentes abastecedoras frente al $\mathrm{CC}$ ha sido analizada bajo diferentes tensores, como el crecimiento poblacional, los cambios en el uso del suelo y la producción hidroeléctrica [5, 6]. Estas investigaciones evidencian que los componentes exposición y sensibilidad son claves para reducir la vulnerabilidad frente al CC [7]; sin embargo, no todas abordan la capacidad adaptativa.

En Colombia, los desarrollos metodológicos para analizar la vulnerabilidad del recurso hídrico ante el CC son escasos [8]. Sin embargo, en el ámbito regional se han desarrollado ejercicios metodológicos en otras temáticas [9-11]. Este documento presenta una propuesta de optimización metodológica para analizar la vulnerabilidad a partir de los lineamientos conceptuales del IPCC con un enfoque de adaptación, retomando el abordaje sistémico planteado por el proyecto AVA [9] a través de dimensiones y el uso de indicadores, y los arreglos matemáticos adelantados por Valencia [12] en su investigación.

Se plantea el abordaje de la vulnerabilidad desde las características particulares de una localidad, para ir avanzando en la interacción con otros elementos que permitan subir la escala de estudio para trabajar la vulnerabilidad desde el territorio hacia lo regional y/o nacional (Bottom-up), favoreciendo la posibilidad de integrar en los procesos de manejo y gestión del recurso hídrico los elementos determinantes en la provisión de agua para consumo humano y, por último, propiciando la transferencia tecnológica por parte de los centros de I+D bajo los términos propuestos por el Panel Intergubernamental sobre Cambio Climático (IPCC).

\section{MATERIALES Y MÉTODOS}

\section{1 Área de estudio}

El Macizo Colombiano es un área estratégica por su diversidad ecosistémica y los múltiples servicios ambientales que ofrece. Aquí se originan ríos como Patía, Magdalena, Putumayo, Caquetá y Cauca. Este último tiene entre sus tributarios el río Las Piedras que suministra agua a la ciudad de Popayán (capital departamental) y el río Palacé que recibe aguas del río Michicao, el cual abastece al municipio de Cajibío (figura 1). La aplicación de la metodología se efectúa sobre dos casos de estudio: la subcuenca Las Piedras y Michicao. 


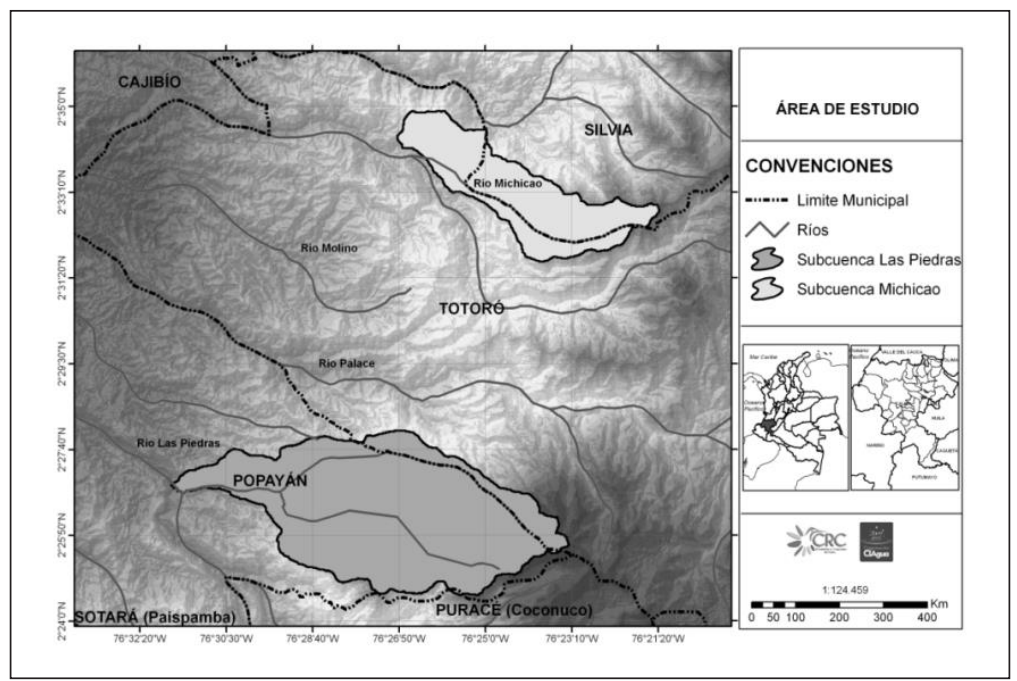

Figura 1. Mapa de las subcuencas Las Piedras y Michicao Fuente: los autores

Los actores sociales de la subcuenca Las Piedras han trabajado, por más de 20 años, en la conservación del agua, el mantenimiento de las coberturas vegetales y la resolución de conflictos territoriales, entre otros procesos. Muestra de ello son los 33 pactos de convivencia, las 54 reservas de la sociedad civil, y numerosas actividades de sostenibilidad desarrolladas por la Fundación Río Las Piedras, Asocampo y las comunidades indígenas de Puracé y Quintana, entre otros [13, 14].

En la subcuenca río Michicao se encuentran comunidades indígenas y campesinas; está ubicada entre los municipios de Totoró y Silvia, pero abastece, aguas abajo, la cabecera del municipio de Cajibío. Esta situación jurisdiccional ha dificultado las actividades de conservación, ya que Totoró y Silvia están limitados para invertir en cuencas que abastecen otro municipio, y Cajibío no puede adelantar acciones por fuera de sus límites territoriales.

\subsection{Propuesta de metodología para el análisis de la vulnerabilidad de fuentes abastecedoras}

Para evaluar la vulnerabilidad frente al CC y la VC en las fuentes abastecedoras, se establece la Unidad Mínima de Análisis (UMA) [9] en función de una escala espacial y los límites político-administrativos o ambientales ( $c f$. Departamento, Municipio, Distrito, Vereda o subcuenca), reconociendo características intrínsecas como los usos del suelo, condiciones climáticas, oferta hídrica, actividades productivas, densidad poblacional, entre otros. 
Se empleó el modelo de análisis DRSPI (fuerza motriz, presión, estado, impacto, respuestas) [15] para establecer las relaciones entre los elementos de la UMA y la identificación de indicadores de vulnerabilidad. Aplicado al contexto de las fuentes abastecedoras, se evidencian las siguientes relaciones: $i$ ) direccionadores naturales y antropogénicos como el cambio y la variabilidad climática, y los patrones de uso del suelo, respectivamente; ii) de presión como la alteración en la frecuencia e intensidad de las lluvias, la demanda de agua para los diferentes usos y la contaminación de esta; iii) de estado, calidad y cantidad del agua; $i v$ ) de impacto, problemas de salud y desabastecimiento de agua; $y, v)$ de respuesta a partir de la regulación, generación y adopción de políticas en torno a la gestión del recurso y cambios en los comportamientos humanos.

Este análisis de vulnerabilidad considera los tres componentes propuestos por el IPCC [3], y se aplican en este estudio, así: i) Exposición: grado en que la UMA se encuentra expuesta a variaciones climáticas extremas, ii) Sensibilidad: grado en el cual un sistema responderá a un cambio dado en el clima, incluyendo efectos benéficos o dañinos y iii) Capacidad de adaptación: Son los medios por los cuales la sociedad usa los recursos disponibles y las habilidades para enfrentar las consecuencias sobre la UMA.

El análisis debe hacerse desde un enfoque sistémico que tenga en cuenta el conjunto de factores extrínsecos (modelos y políticas de desarrollo nacional e internacional, cambio global) e intrínsecos (políticas locales, saberes tradicionales, tipo de ecosistema y patrones culturales) que determinan la vulnerabilidad de los sistemas considerados. A continuación, se describen los pasos propuestos para el análisis y los diferentes momentos de realimentación con los actores sociales.

\section{Paso 1: Delimitación de las características del modelo}

Para delimitar el alcance del análisis de vulnerabilidad, se debe dar respuesta a los siguientes interrogantes en torno a los atributos de la vulnerabilidad:

- ¿Quién o qué es vulnerable? - Unidad mínima de análisis (subcuenca).

- ¿Frente a qué se es vulnerable? -Definición de la amenaza (variación climática y eventos extremos).

- ¿Cuándo o en qué momento es vulnerable?- Horizonte temporal (vulnerabilidad actual - a variabilidad climática y futura a cambio climático).

- ¿Cuál es el atributo específico de la vulnerabilidad?- cantidad y calidad del agua.

Paso 2: Definición de dimensiones en la UMA:

a) Biofísica, hace referencia a un conjunto de estructuras y relaciones en el contexto biótico y abiótico. 
34 M. P. Valencia R. •A. Figueroa C.•D. M. Ruiz O.•J.D. Otero S. •J. P. Martínez I. V. E. Ceballos S. • S. C. Joaquí D. • D. González Q.

b) Político-institucional, tiene en cuenta la capacidad de gestión institucional, administrativa y fiscal de las instituciones en los niveles local, regional y nacional.

c) Económico-productiva: contempla las dinámicas económicas de los productos y servicios relacionados con la UMA

d) Socio-cultural: comprende las características e interacciones de las comunidades humanas en el interior de la UMA.

\section{Paso 3: Selección de indicadores}

Se seleccionan teniendo en cuenta argumentos inductivos, es decir, que se dan sobre la base de datos o información disponible de variables medidas en la zona que pueden obtenerse a través de información cuantitativa y cualitativa, y se clasifican teniendo en cuenta los criterios descritos por Niemeijer [15] en torno a la pertinencia, disponibilidad, accesibilidad, medible, único y especificidad de los indicadores.

El listado final de indicadores se elaboró según los componentes de la vulnerabilidad (sensibilidad, exposición o capacidad adaptativa) del sistema estudiado. Para garantizar su comparación se normalizan los datos a través de la fórmula empleada por González y otros [16] en un rango de 0 y 1 . Los resultados se relacionan en la tabla 1.

Tabla 1. Indicadores seleccionados

\begin{tabular}{|c|c|c|c|c|}
\hline \multirow[t]{2}{*}{ Dimension } & \multirow[t]{2}{*}{ Indicadores (unidades) } & $\begin{array}{l}\text { Rio Las } \\
\text { Piedras }\end{array}$ & $\begin{array}{c}\text { Rio } \\
\text { Michicao } \\
\end{array}$ & \multirow[t]{2}{*}{ Fuente de información } \\
\hline & & Valor $(x)$ & Valor $(x)$ & \\
\hline \multirow{4}{*}{ Biofísica } & $\begin{array}{l}\text { Índice de regulación hídrica } \\
\text { (unidad) }\end{array}$ & 0.70 & 0.57 & ENA 2010 \\
\hline & $\begin{array}{l}\text { Coberturas Reguladoras del } \\
\text { ciclo Hidrológico (unidad) }\end{array}$ & 3885.3 & 1295 & SIG. CIAgua \\
\hline & Índice de Uso de Agua (unidad) & 35.00 & 0.60 & ENA 2010 \\
\hline & $\begin{array}{l}\text { Índice de fragmentación } \\
\text { (unidad) }\end{array}$ & 338.16 & 160.86 & SIG. CIAgua. \\
\hline \multirow{4}{*}{ Biofísica } & $\begin{array}{l}\text { Índice de calidad de agua } \\
\text { (unidad) }\end{array}$ & 82.00 & 63.44 & CIAgua \\
\hline & Precipitación (mm) & 1926 & 1711 & $\begin{array}{l}\text { Proyecto Acueducto 2012. POT } \\
\text { Silvia y Totoró }\end{array}$ \\
\hline & Caudal (m/s) & 2.60 & 0.30 & $\begin{array}{l}\text { POMCH Río Las Piedras. Pla- } \\
\text { nes de ahorro y uso eficiente del } \\
\text { agua 2012. APC Cajibío }\end{array}$ \\
\hline & Temperatura $\left({ }^{\circ} \mathrm{C}\right)$ & 19.30 & 14.00 & $\begin{array}{l}\text { POMCH. Río Las Piedras. Plan } \\
\text { de desarrollo Totoró 2012-2015 }\end{array}$ \\
\hline
\end{tabular}




\begin{tabular}{|c|c|c|c|c|}
\hline \multirow[t]{2}{*}{ Dimension } & \multirow[t]{2}{*}{ Indicadores (unidades) } & $\begin{array}{l}\text { Rio Las } \\
\text { Piedras }\end{array}$ & $\begin{array}{c}\text { Rio } \\
\text { Michicao }\end{array}$ & \multirow[t]{2}{*}{ Fuente de información } \\
\hline & & Valor $(x)$ & $\operatorname{Valor}(x)$ & \\
\hline \multirow{2}{*}{$\begin{array}{l}\text { Político } \\
\text { institutional }\end{array}$} & $\begin{array}{l}\text { Inversión del acueducto en } \\
\text { gestión ambiental para la cuenca } \\
(\%)\end{array}$ & 45.00 & 0.00 & $\begin{array}{l}\text { Plan de desarrollo Popayán } \\
\text { 2012-2015. Fundación Río Las } \\
\text { Piedras. APC de Cajibío }\end{array}$ \\
\hline & $\begin{array}{l}\text { Actores relacionados con el } \\
\text { recurso hídrico en la cuenca (\#) }\end{array}$ & 12.00 & 6.00 & Talleres CIAgua \\
\hline \multirow{2}{*}{$\begin{array}{l}\text { Económico } \\
\text { productiva }\end{array}$} & $\begin{array}{l}\% \text { Cobertura acueducto- rural } \\
(\%)\end{array}$ & 50.00 & 29.60 & $\begin{array}{l}\text { Informe de Gestión. Datos bá- } \\
\text { sicos del suministro de agua y } \\
\text { servicio de alcantarillado, } 2012 . \\
\text { PBOT, } 2002\end{array}$ \\
\hline & $\begin{array}{l}\% \text { Cobertura acueducto - urba- } \\
\text { na (\%) }\end{array}$ & 99.50 & 96.30 & $\begin{array}{l}\text { Informe de Gestión. Datos bá- } \\
\text { sicos del suministro de agua y } \\
\text { servicio de alcantarillado, } 2012 . \\
\text { PBOT, } 2002\end{array}$ \\
\hline \multirow{4}{*}{$\begin{array}{l}\text { Socio } \\
\text { cultural }\end{array}$} & $\begin{array}{l}\text { Densidad poblacional rural } \\
\text { (unidad) }\end{array}$ & 19.47 & 79.65 & DANE, 2005-CIAgua \\
\hline & $\begin{array}{l}\text { Densidad poblacional urbana } \\
\text { (unidad) }\end{array}$ & 7.75 & 4.89 & DANE, 2005-CIAgua \\
\hline & $\begin{array}{l}\text { Percepción de eficiencia del } \\
\text { servicio de acueducto usuarios } \\
\text { zona urbana (\%) }\end{array}$ & 79.60 & 67.00 & Talleres CIAgua \\
\hline & $\begin{array}{l}\text { Percepción de eficiencia del } \\
\text { recurso hídrico por usuarios } \\
\text { zona rural (\%) }\end{array}$ & 63.30 & 62.50 & Talleres CIAgua \\
\hline
\end{tabular}

Fuente: los autores

Paso 4. Fórmula para el análisis de la vulnerabilidad

Partiendo de que la vulnerabilidad $(V)$ se encuentra en función de la exposición (ex), la sensibilidad (se) y la capacidad adaptativa $(c a)$, y que se expresa como:

$$
V=e x+s e-c a
$$

Se procede a calcular cada uno de los componentes (ex, se, ca) teniendo en cuenta el valor ponderado de cada indicador $(p n)$ y su relevancia o peso (wn) en la vulnerabilidad del sistema, como se muestra en las siguientes ecuaciones:

$$
e x=\sum_{i=1}^{n} I_{i}=I_{(p 1)(w 1)}+I_{(p 2)(w 2)}+I_{(p 3)(w 3)}+I_{n}=1
$$




$$
\begin{aligned}
& s e=\sum_{i=1}^{n} I_{i}=I_{(p 1)(w 1)}+I_{(p 2)(w 2)}+I_{(p 3)(w 3)}+I_{n}=1 \\
& c a=\sum_{i=1}^{n} I_{i}=I_{(p 1)(w 1)}+I_{(p 2)(w 2)}+I_{(p 3)(w 3)}+I_{n}=1
\end{aligned}
$$

Donde: $V=$ vulnerabilidad, ex= exposición, $s e=$ sensibilidad, $c a=$ capacidad adaptativa,

$\mathrm{p}=$ indicador ponderado, $\mathrm{w}=$ peso o valor de influencia del indicador

Teniendo en cuenta que ex, se, y ca pueden tener un valor máximo de 1 , entonces la vulnerabilidad puede variar entre $-1 \geq V \geq 2$. No obstante, para una mejor interpretación de los valores a través de una escala de 0 a 1 , se aplica una redistribución a los rangos teniendo en cuenta la siguiente expresión matemática:

$$
V=e x+s e-c a+\frac{1}{3}
$$

De este modo, cada uno de los componentes del modelo puede tener en la fórmula general de vulnerabilidad valores entre el 0 y el 1 , en donde el 0 se asocia a condiciones de baja exposición y baja sensibilidad; y los valores cercanos al 1 se manejan de manera opuesta. Según lo anterior, la vulnerabilidad tiene seis rangos equidistantes de interpretación como se muestra en la figura 2.

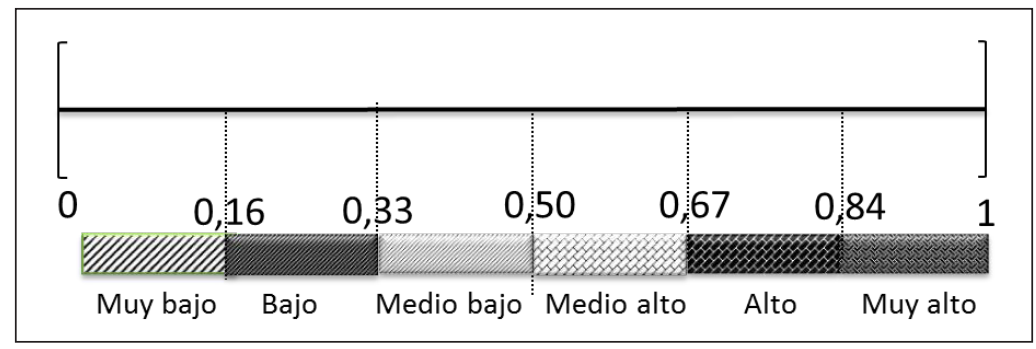

Figura 2. Rangos propuestos para la interpretación de la vulnerabilidad Fuente: los autores

El procedimiento de cálculo para cada componente de la vulnerabilidad se hizo a través de un análisis matricial, de acuerdo con los datos existentes para el territorio estudiado y teniendo en cuenta que algunos de los indicadores utilizados se comportan de manera inversa, es decir, son más sensibles, expuestos y con una mayor capacidad adaptativa cuando se acercan a 0 (y no cuando se aproximan a 1 como se esperaría), 
como es el caso de índice de fragmentación, coberturas reguladoras del ciclo hidrológico, índice de calidad de agua, temperatura, percepción de eficiencia del servicio de acueducto por usuarios de la zona urbana, percepción de eficiencia del recurso hídrico por usuarios zona rural.

\section{Paso 5. Evaluación de la vulnerabilidad actual y futura}

Empleando datos históricos y/o actuales para cada uno de los indicadores, se efectuó el cálculo de la vulnerabilidad por medio de matrices matemáticas en el caso de que los datos sean únicos, puntales o que estén atribuidos a un polígono, o de acuerdo con la cantidad de datos pueden emplearse programas de sistemas de información geográfica para trabajar a través de álgebra de mapas la interacción de la información raster (celdas o píxeles de información).

De acuerdo con los anteriores elementos y conociendo las tendencias de los indicadores priorizados para cada escenario, es posible obtener proyecciones bajo diferentes niveles de presión sobre el territorio, ocasionados por las variaciones climáticas, los fenómenos naturales o por acciones humanas que modifican directamente el comportamiento de indicadores de las dimensiones integradas en el modelo. Se plantean escenarios de cambio, los cuales responden a tendencias bajo porcentajes de variación específicos (tomados de las fuentes de información o conocimiento de expertos) y generan ventanas de cambio moderadas u optimistas (cambios leves) y severas o pesimistas (cambios severos). Los escenarios planteados se relacionan en la tabla 2.

Tabla 2. Descripción de los escenarios de análisis propuestos

\begin{tabular}{|l|l|}
\hline \multicolumn{1}{|c|}{ Escenario } & \multicolumn{1}{c|}{ Descripción } \\
\hline Presente & $\begin{array}{l}\text { Basada en el comportamiento normal de cada subcuenca, sin incidencia de eventos climáticos } \\
\text { extremos }\end{array}$ \\
\hline $\begin{array}{l}\text { Niño } \\
\text { Moderado }\end{array}$ & $\begin{array}{l}\text { Presenta una variabilidad climática moderada con incidencia en el componente de exposición, } \\
\text { disminuyendo el caudal y la precipitación, mientras aumenta la temperatura. Las otras variables } \\
\text { tienen un comportamiento normal de cada subcuenca }\end{array}$ \\
\hline $\begin{array}{l}\text { Niña } \\
\text { Moderado }\end{array}$ & $\begin{array}{l}\text { Presenta una variabilidad climática moderada con incidencia en el componente de exposición, } \\
\text { aumentando el caudal y la precipitación, mientras disminuye la temperatura. Las otras variables } \\
\text { continúan con el comportamiento normal }\end{array}$ \\
\hline $\begin{array}{l}\text { Con baja } \\
\text { capacidad } \\
\text { adaptativa } \\
\text { (BCA) }\end{array}$ & $\begin{array}{l}\text { Se considera una disminución en los indicadores del componente de capacidad adaptativa: Inver- } \\
\text { sión del acueducto en gestión ambiental para la cuenca, actores relacionados con el recurso hídrico, } \\
\text { \% Cobertura acueducto rural y urbano. Los anteriores cambios inciden sobre otras variables del } \\
\text { modelo disminuyendo las coberturas reguladoras del ciclo hidrológico, el índice de calidad de } \\
\text { agua, la percepción de eficiencia del servicio de acueducto usuarios zona urbana y la percepción } \\
\text { de eficiencia del recurso hídrico por usuarios zona rural. También incrementando los Índices de } \\
\text { fragmentación y de uso de Agua, la densidad poblacional rural y urbana }\end{array}$ \\
\hline
\end{tabular}


38 M. P. Valencia R. A. Figueroa C.•D. M. Ruiz O.•J. D. Otero S. J. P. Martínez I. •V. E. Ceballos S. S. C. Joaquí D. •D. González Q.

\begin{tabular}{|l|l|}
\hline \multicolumn{1}{|c|}{ Escenario } & \multicolumn{1}{c|}{ Descripción } \\
\hline $\begin{array}{l}\text { Con alta } \\
\text { capacidad } \\
\text { adaptativa } \\
\text { (ACA) }\end{array}$ & $\begin{array}{l}\text { Se considera un aumento en los indicadores del componente de capacidad adaptativa: Inversión } \\
\text { del acueducto en gestión ambiental para la cuenca, actores relacionados con el recurso hídrico } \\
\text { en la cuenca, \% cobertura acueducto rural y urbano. Estos cambios afectan otras variables del } \\
\text { modelo incrementando las coberturas reguladoras del ciclo hidrológico, el índice de calidad de } \\
\text { agua, la percepción de eficiencia del servicio de acueducto usuarios zona urbana, la percepción } \\
\text { de eficiencia del recurso hídrico por usuarios zona rural, la densidad poblacional rural y urbana. } \\
\text { También se disminuyen los índices de fragmentación y de uso de Agua }\end{array}$ \\
\hline
\end{tabular}

Fuente: los autores

\section{RESULTADOS Y DISCUSÓN}

\subsection{Escenarios de vulnerabilidad subcuenca río Las Piedras}

\section{a) Actual}

La subcuenca Las Piedras en el escenario actual presentó una vulnerabilidad media baja. Esta condición es producto de la capacidad adaptativa atribuida a los procesos sinérgicos desarrollados por los actores locales. El fortalecimiento social ha permitido la conservación y recuperación de las zonas de regulación hídrica involucrando a las comunidades mediante alternativas productivas sostenibles e inversión directa. Adicionalmente, la planificación ambiental participativa integra acciones complementarias, generando mejores condiciones biofísicas en la subcuenca que reducen la sensibilidad a eventos climáticos moderados y garantizan un caudal permanente que mantiene el abastecimiento hídrico (tabla 3).

Tabla 3. Resultados escenarios subcuenca río Las Piedras

\begin{tabular}{|c|c|c|c|c|c|}
\hline Escenarios & $S$ & $E$ & $C A$ & $V$ & Vulnerabilidad \\
\hline Presente & 0.32 & 0.52 & 0.57 & 0.42 & I \\
\hline Niño moderado & 0.32 & 0.64 & 0.57 & 0.46 & I \\
\hline Niña moderada & 0.37 & 0.56 & 0.57 & 0.45 & I \\
\hline Niño moderado BCA & 0.44 & 0.64 & 0.35 & 0.57 & I \\
\hline Niña moderada BCA & 0.45 & 0.56 & 0.37 & 0.55 & I \\
\hline Niño moderado ACA & 0.30 & 0.64 & 0.71 & 0.41 & I \\
\hline Niña moderada ACA & 0.32 & 0.56 & 0.71 & 0.39 & I \\
\hline
\end{tabular}

Fuente: los autores

b) Fenómeno de El Niño moderado, con alta y baja capacidad adaptativa

La vulnerabilidad para el escenario Niño moderado es media baja $(0,46)$, producto de la reducción del caudal que incrementa la exposición de la subcuenca. Adicionalmente, la ampliación de las áreas productivas favorece la contaminación del suelo, del agua y la fragmentación ecosistémica [17] incrementando la sensibilidad. 
En el escenario Niño moderado con baja capacidad adaptativa (negativo), al disminuir el caudal y reducir la inversión del acueducto en la subcuenca, se limita el acceso de las comunidades al agua potable. En este sentido, la pérdida de la capacidad adaptativa y el aumento de la sensibilidad incrementan la exposición del sistema a la VC generando condiciones de desabastecimiento y una vulnerabilidad media-alta $(0,57)$.

Como escenario positivo, el Niño moderado con alta capacidad adaptativa incorpora procesos participativos, con metas comunes en diferentes niveles, encaminadas a mejorar la gestión ambiental de la subcuenca interviniendo de forma sinérgica en todos los componentes. Al aumentar la inversión en la subcuenca, se establecen sistemas de producción sostenibles y zonas de recuperación ecológica que mejoran la regulación hídrica en la UMA; esto mejora el acceso al recurso hídrico y favorece la percepción de los usuarios.

En este último escenario, al incrementar la capacidad adaptativa, se presenta una incidencia positiva sobre los indicadores del componente de sensibilidad de la subcuenca, disminuyendo la vulnerabilidad.

\section{c) Fenómeno de La Niña, moderado, con alta y baja capacidad adaptativa}

El escenario Niña moderado presenta una vulnerabilidad media- baja $(0,45)$, producto de la capacidad de regulacion del sistema y el incremento en la precipitación que favorece la oferta hídrica.

Para el escenario Niña moderado con baja capacidad adaptativa la vulnerabilidad es 0,55 (medio alta). Las proyecciones que causan estos resultados están relacionadas con la desarticulación de actores y la disminución en las inversiones institucionales (capacidad adaptativa), que impactan la dimensión biofísica y limitan la disponibilidad del agua.

Bajo un escenario de Niña moderado con alta capacidad adaptativa la vulnerabilidad es medio baja $(0,39)$ como resultado del fortalecimiento del tejido social y las acciones de gestión interinstitucional que mejoran la regulación hídrica en la cuenca, al favorecer el cambio del uso del suelo relacionado con actividades productivas y de conservación.

\subsection{ESCENARIOS DE VULNERABILIDAD SUBCUENCA RÍO MICHICAO}

a) Actual

Para la subcuenca del río Michicao en el escenario actual, la vulnerabilidad media alta $(0,54)$ es atribuida a la desarticulación social, la baja presencia institucional y 
la escasa inversión ambiental (tabla 4). La capacidad adaptativa se afecta por los conflictos jurisdiccionales y una baja cobertura del acueducto en el municipio de Cajibío. Las limitaciones en la planificación ambiental dificultan el desarrollo de programas que conserven las zonas de regulación hídrica de la subcuenca.

Tabla 4. Resultados escenarios subcuenca río Michicao

\begin{tabular}{|c|c|c|c|c|c|}
\hline Escenarios & S & E & CA & V & Vulnerabilidad \\
\hline Presente & 0.46 & 0.51 & 0.36 & 0.54 & I \\
\hline Niño moderado & 0.46 & 0.77 & 0.36 & 0.63 & I \\
\hline Niña moderada & 0.48 & 0.56 & 0.36 & 0.56 & I \\
\hline Niño moderado BCA & 0.56 & 0.77 & 0.24 & 0.70 & $\mathrm{I}$ \\
\hline Niña moderada BCA & 0.55 & 0.57 & 0.25 & 0.62 & $\mathrm{I}$ \\
\hline Niño moderado ACA & 0.46 & 0.77 & 0.51 & 0.58 & $\mathrm{I}$ \\
\hline Niña moderada ACA & 0.42 & 0.57 & 0.51 & 0.49 & \\
\hline
\end{tabular}

Fuente: los autores

Sumado a lo anterior, las condiciones biofísicas existentes en la UMA, como la fragmentación de coberturas vegetales, alta presión sobre el recurso hídrico y una baja calidad del recurso (ICA) en la zona, aumentan la sensibilidad del sistema ante fenómenos climáticos, fenómeno de El Niño moderado, con alta y baja capacidad adaptativa.

\section{b) Fenómeno de El Niño moderado, con alta y baja capacidad adaptativa}

El escenario de Niño moderado presenta aumento de temperatura y disminución de precipitación y caudal (exposición), empleando proyecciones referidas en estudios de VC y CC, en los niveles local, nacional e internacional [3, 10, 18-20]. La vulnerabilidad aquí es media- alta, dada la inequidad en el acceso al agua potable en Cajibío, especialmente en la zona media-baja del municipio donde el servicio es intermitente. La presión respecto a la oferta disponible en eventos de Niño moderado es alta, debido a la demanda para las actividades productivas y de consumo doméstico, contribuyendo con la reducción de caudales y la capacidad de adaptación del socioecosistema.

Considerando las anteriores modificaciones, se generó el escenario niño moderado con baja capacidad adaptativa (tabla 2), donde se disminuyeron los indicadores de capacidad adaptativa, que inciden también sobre el componente de sensibilidad y queda el sistema más expuesto a la variabilidad climática

Contrario a lo anterior, se obtuvo un análisis de Niño moderado con alta capacidad adaptativa. Bajo este escenario, se favorecen acciones que benefician notablemente las cuencas abastecedoras de agua, debido principalmente a la mayor inversión en la 
subcuenca, lo cual le permite involucrar y articular actores sociales e institucionales en torno a la subcuenca.

\section{c) Fenómeno de La Niña moderado, con alta y baja capacidad adaptativa}

El escenario de Niña moderado (tabla 2) indicó que este sistema responderá de la misma manera al escenario actual, dada la similitud en los valores de los indicadores de capacidad adaptativa y sensibilidad, limitando la incidencia de la exposición en la fórmula de vulnerabilidad.

Considerando las modificaciones ya descritas, se generó el escenario con baja capacidad adaptativa (tabla 2). La regulación hídrica presenta una retención y regulación de humedad media; el uso de agua aumenta por la oferta disponible y la calidad de agua disminuye por el aumento en los procesos de erosión y sedimentación en la cuenca. La reducción de las coberturas reguladoras del ciclo hidrológico disminuyó el caudal de los afluentes, y afectó la oferta de agua para el acueducto, los porcentajes de cobertura del acueducto (rural y urbano) y la percepción de la eficiencia del servicio de acueducto por parte de los usuarios.

El escenario con alta capacidad adaptativa aumentó la inversión municipal para la cuenca, y favoreció las coberturas reguladoras del ciclo hidrológico por procesos de reforestación y la disminución de la erosión hacía el cauce principal. El aumento del porcentaje de cobertura de acueducto rural y urbano creó un ambiente de satisfacción reflejado en la percepción de los usuarios del acueducto.

\section{CONCLUSIONES}

La metodología propuesta permite analizar, de forma sistémica, la vulnerabilidad de las fuentes de abastecimiento frente a la variabilidad y al cambio climático, mediante la inclusión de indicadores de diferentes dimensiones que resumen las características socioecosistémicas del territorio. Se considera que la metodología puede ser replicada en otros sistemas de interés para análisis similares.

La vulnerabilidad de las subcuencas en el escenario actual es media-alta para Michicao y medio baja para Las Piedras. Estos resultados están relacionados con la gobernanza local sobre el recurso hídrico, la cual determina el estado de conservación de las subcuencas hidrográficas y la capacidad de adaptativa social e institucional ante la variabilidad climática para garantizar el abastecimiento de agua.

La capacidad adaptativa es dinámica en cada territorio y depende de una gestión que interrelacione aspectos económicos, naturales, institucionales, tecnológicos y de organización social. La subcuenca del río Las Piedras lleva más de 20 años fortaleciendo la planificación integral participativa, en torno al manejo de agua considerando 
estrategias de reducción de riesgos climáticos; contrario a lo que sucede en la subcuenca Michicao donde la capacidad adaptativa está limitada por la jurisdicción municipal y la ausencia de un mecanismo de articulación de acciones, inversiones y actores en torno a la gestión de las fuentes abastecedoras.

\section{AGRADECIMIENTOS}

Este estudio se desarrolló gracias al convenio de cooperación 0201-2013 celebrado entre CRC y CIAgua y la participación de las comunidades campesinas, administraciones locales, cabildos indígenas, Universidad del Cauca, grupos de investigación GEA y GIT, entidades territoriales y empresas de acueducto de los municipios de Silvia, Totoró, Cajibío y Popayán.

\section{REFERENCIAS}

[1] IDEAM, Colombia Segunda Comunicación Nacional ante la Convención Marco de las Naciones Unidas sobre el Cambio Climático. Bogotá: IDEAM, 2010.

[2] IPCC, «Summary for Policymakers. In: Managing the Risks of Extreme Events and Disasters to Advance Climate Change Adaptation», in A Special Report of Working Groups I and II of the Intergovernmental Panel on Climate Change, C. B. Field, et al., Eds., ed Inglaterra: Cambridge University Press, Cambridge, UK, 2012, p. 32.

[3] IPCC, “Cambio climático 2007: Informe de síntesis. Contribución de los Grupos de trabajo I, II y III al Cuarto Informe de evaluación del Grupo Intergubernamental de Expertos sobre el Cambio Climático", ed. Ginebra: Grupo Intergubernamental de Expertos sobre el Cambio Climático. http://www.ipcc.ch/pdf/assessment-report/ar4/syr/ar4_syr_sp.pdf, 2007.

[4] J. W. D. Handmer, Steven.Downing, Thomas E. «Societal vulnerability to climate change and variability». Mitigation and Adaptation Strategies for Global Change, vol. 4, pp. 267-281, 1999.

[5] S. A. M. Vaghefi, SJ.Abbaspour, KC. Srinivasan, R. Arnold, JR., "Integration of hydrologic and water allocation models in basin-scale water resources management considering crop pattern and climate change: Karkheh River Basin in Iran". Regional Environmental Change, vol. 13, pp. 1-10, 2012.

[6] M. Gaál, Quiroga, Sonia, Fernández-Haddad, Zaira, "Potential impacts of climate change on agricultural land use suitability of the Hungarian counties". Regional Environmental Change, vol. 14, p. 13, 2014.

[7] K. A. Nicholas and W. H. Durham, "Farm-scale adaptation and vulnerability to environmental stresses: Insights from winegrowing in Northern California". Global Environmental Change, vol. 22, pp. 483-494, 2012.

[8] IDEAM, Estudio Nacional del Agua 2010. Bogotá D. C.: Instituto de Hidrología, Meteorología y Estudios Ambientales, 2010.

[9] A. Figueroa, et al., "Inter-Institutional, Multi-Sectoral Analysis of Vulnerability and Adapta- 
tion to Climate Change for the Agricultural Sector in the Upper Cauca River Basin Impacting Adaptation Policies. Final report”, Popayán2013.

[10] IDEAM, "Metodologia para el análisis de vulnerabilidad y análisis de riesgos asociados en la cuenca alta del río Cauca. Programa de Integración de ecosistemas y Adaptación al Cambio Climático en el Macizo Colombiano". Instituto de Hidrología, Metereología y Estudios Ambientales, vol. I, 2011.

[11] PNUD, «Analisis de vulnerabilidad y adaptacion a cambio climatico para la subcuenca rio Molino (parte alta y media), municipio de Popayan, Cauca». Acueducto y Alcantarillado de Popayán SA-ESP (AAPSA), Popayán2011.

[12] M. Valencia, «Suceptibilidad de las lagunas de páramo a procesos degradativos de origen antrópico incorporando la complejidad ambiental del territorio (departamento del Cauca, Colombia)». Tesis Doctoral. Doctorado Interintitucional en Ciencias Ambientales, Universidad del Cauca, Popayán, (en prensa).

[13] CRC, «Plan de ordenación y manejo de la subcuenca hidrográfica del río las piedras. Corporación Autónoma Regional del Cauca (CRC)», 2006.

[14] L. Recaman, «Planificación ambiental como estrategia para la conservación de una fuente de abastecimiento». Ambiente y Sostenibilidad, pp. 50-58, 2012.

[15] D. Niemeijer and R. S. de Groot, «A conceptual framework for selecting environmental indicator sets». Ecological Indicators, vol. 8, pp. 14-25, 2008.

[16] C. G. González, et al., Tratamiento de datos vol. 150: Ediciones Díaz de Santos, 2006.

[17] M. Ordoñez and A. Figueroa, «Estudio espacio-temporal del proceso de fragmentación sobre las coberturas boscosas en la cuenca del río Palacé», in Fragmentación y Coberturas Vegetales en Ecosistemas Andinos, Departamento del Cauca, A. Figueroa and M. Valencia, Eds., ed Popayán: Universidad del Cauca, 2009, pp. 247-266.

[18] J. F. Casanova, «Determinación de un régimen de caudal ambiental utilizando una aproximación a la metodología para proyectos licenciados de Colombia en la subcuenca del río las Piedras en el departamento del Cauca» Tesis de pregrado. Programa de Ingeniería Ambiental, Universidad del Cauca, 2012.

[19] EMCASERVICIOS, «Acueducto Michicao. Municipio de Cajibío. Departamento del Cauca: Análisis y estudio de alternativas», Empresa Caucana de Servicios Públicos S. A. ESP. 2010.

[20] GEA, «Modelamiento climatico, patrones de cambio y sus efectos en ecosistemas agricolas altoandinos, identificando medidas de adaptacion y mitigacion para la planificacion.,» Universidad del Cauca - Ministerio de Agricultura y Desarrollo Rural Popayán, Programa de Investigación 2012 . 